\title{
Costs and Resource Utilization Among Medicaid Patients with Schizophrenia Treated with Paliperidone Palmitate or Oral Atypical Antipsychotics
}

\author{
Jacqueline A. Pesa ${ }^{1} \cdot$ Erik Muser $^{1} \cdot$ Leslie B. Montejano $^{2}$. \\ David M. Smith ${ }^{2} \cdot$ Oren I. Meyers $^{2}$
}

Published online: 13 October 2015

(c) The Author(s) 2015. This article is published with open access at Springerlink.com

\begin{abstract}
Background Non-adherence to antipsychotic therapy among patients with schizophrenia is a key driver of relapse, which can lead to costly inpatient stays. Longacting injectables (LAIs) may improve adherence, thus reducing hospitalizations, but inpatient cost reductions need to be balanced against higher drug acquisition costs of LAIs. Real-world evidence is needed to help quantify the economic value of oral atypical antipsychotics compared with LAIs.

Objective The objective of this study was to compare healthcare costs and resource utilization between oncemonthly paliperidone palmitate (PP) and oral antipsychotic therapy (OAT) in a population of Medicaid beneficiaries with schizophrenia.

Methods A retrospective, observational study was performed using Truven Health MarketScan Medicaid claims data from 2009 to 2012. Marginal structural modeling, a form of weighted repeated measures analysis to control for differences between cohorts and time-varying confounding, was used to estimate monthly costs of care in 2012 US dollars and resource utilization over a 12-month period for patients in each cohort.

Results While per-month mental-health prescription costs were US\$1019 higher in the PP cohort, approximately
\end{abstract}

Jacqueline A. Pesa

jpesa@its.jnj.com

1 Janssen Scientific Affairs, LLC, 3133 Castle Peak Ave. Superior, Louisville, CO 80027, USA

2 Truven Health Analytics, Cambridge, USA
$55 \%$ of this premium was offset by lower inpatient and outpatient care costs, producing a mean monthly total cost differential of US\$434 (95\% CI 298-569, $p<0.0001)$ for all-cause costs and US\$463 (95\% CI 374-552, $p<0.0001)$ for mental-health-related costs. Use of PP also resulted in a 0.44 and 0.47 reduction in the odds of allcause and mental-health-related hospitalizations and a 0.09 reduction in the odds of all-cause emergency department visits $(p<0.0001, p<0.0001$, and $p=0.0134$, respectively) over the 12-month follow-up period.

Conclusions Treatment with long-acting injectable antipsychotics, such as PP, may reduce inpatient and outpatient healthcare services utilization and associated costs. These findings also suggest that patients with schizophrenia taking once-monthly PP may stand a lower risk of hospitalization than patients on OAT.

\section{Key Points}

While the paliperidone palmitate (PP) cohort had higher mental-health prescription costs compared with the oral antipsychotic therapy (OAT) cohort, over half of the acquisition costs were offset by lower inpatient and outpatient care costs.

PP use showed reductions in the odds of inpatient and outpatient healthcare utilization and associated costs over the 12-month follow-up period.

The study suggests that patients with schizophrenia taking once-monthly PP may experience less inpatient and outpatient healthcare services utilization and associated costs than patients taking OAT. 


\section{Introduction}

Schizophrenia is a chronic, complex, and devastating mental illness, long recognized as costly and challenging to treat [1-3]. The economic impact of schizophrenia attributed to direct healthcare costs alone was estimated at US\$22.7 billion in the United States (2002 US dollars) [3]. Estimates of mean annual direct medical costs among community-dwelling patients with schizophrenia range from US $\$ 3700$ to US $\$ 27,900$ per patient; inpatient costs comprise $10-30 \%$ of these costs [4]. In a recent study of schizophrenia patients with Medicaid coverage, annual mental-health-related medical costs for patients prescribed atypical antipsychotics were US $\$ 11,700$ per patient [5].

Relapse associated with schizophrenia is a key driver of costs [6], principally due to the need for inpatient care. Moreover, as the number of patient relapses increases, inpatient costs disproportionally accelerate [7]; thus, relapse prevention is a key goal in effectively managing schizophrenic patient populations [8]. A significant driver of relapse and subsequent hospitalization or acute care is the frequent non-adherence to antipsychotic therapy among patients with schizophrenia [9]. In fact, a recent estimate provided by the US Department of Health and Human Services revealed that just $61 \%$ of Medicaid patients with schizophrenia continuously refilled their antipsychotic prescriptions [10]. This underscores the need for therapies that may improve adherence, such as long-acting injectable (LAI) antipsychotics, which have demonstrated enhanced adherence [11], thus potentially reducing rates of relapse and hospitalization among patients with schizophrenia [12]. This, however, needs to be balanced against higher drug acquisition costs as well as the cost of administration often associated with LAIs [13].

Paliperidone palmitate long-acting injection (PP) is indicated for the treatment of schizophrenia in adults and has been available in the US since 2009. PP is an injectable antipsychotic medication administered monthly by a healthcare professional, which differs from oral antipsychotic therapy (OAT) that requires patients to take medication daily. There is a body of literature to date demonstrating improved economic outcomes for patients treated with PP; however, most of these studies are either from clinical trials [14] or economic models [15-17]. A recent study of Veterans Affairs patients with schizophrenia treated with PP compared with those treated with OAT showed lower inpatient admission rates and costs for the PP-treated group over the observed 1-year follow-up period [18]. A study in a Medicaid sample recently demonstrated a lower likelihood of emergency department visits among patients treated with PP [11]. A meta-analysis study also suggested that LAIs are associated with greater reductions in hospitalization rates compared with OATs [19]. There is a need for additional real-world evidence accumulated through comparative outcomes studies, to help inform formulary and policy decision makers about the value of LAIs compared with oral atypical antipsychotics for the treatment of schizophrenia. The present study was designed to build upon previous research, especially by expanding the size of the data set and increasing the geographic dispersion of the sample in the US. The primary objective of the study was to compare costs and resource utilization among Medicaid patients diagnosed with schizophrenia who were treated with either PP or OAT in a real-world setting.

\section{Methods}

This study retrospectively compared costs and resource utilization among Medicaid patients diagnosed with schizophrenia who were treated with PP versus OAT. Specifically, PP and OAT patients were compared with respect to demographic and clinical characteristics in the 6 months prior to initiation (baseline or run-in period) of PP or OAT and the healthcare utilization and costs in the baseline period and 12-month post-initiation period (follow-up). Healthcare utilization and direct healthcare costs were analyzed with inverse probability of treatment weights (IPTW) and marginal structural modeling (MSM) to estimate these outcomes while controlling for imbalances in covariates between the PP and the OAT cohorts, including demographic and clinical characteristics and events, such as treatment decisions, that occur not only at index but over time.

\subsection{Study Sample}

Patients in this study were identified from the MarketScan ${ }^{\circledR}$ Medicaid Multi-State Database (Truven Health Analytics, Ann Arbor, MI, USA). This database contains the deidentified healthcare claims of Medicaid enrollees from twelve geographically diverse states. Patients who met the inclusion and exclusion criteria for either the PP or OAT cohort, detailed below, were included in the study and followed over 12 months.

The PP cohort included patients with two or more prescription drug or medical claims for PP, no more than 90 days apart, during the cohort selection period of July 2009 through December 2011. The earliest such claim was set as the index date (day 1). Because of the US launch date for PP (August 2009), all patients were newly started on PP as of the index date. Patients were required to have continuous enrollment 6 months before and 12 months after 
the index date (the 'study period'), be at least 18 years old at index, and have evidence of schizophrenia during the study period, defined as two or more claims with ICD-9 295.1x, 295.2x, 295.3x, 295.6x, 295.8x, or 295.9x, or one or more claim with ICD-9 $295.7 \mathrm{x}$ plus one or more claim with ICD-9 295.1x, 295.2x, 295.3x, 295.6x, 295.8x, or 295.9x. In order to ensure that medication claims histories were as complete as possible, patients with Medicare dual eligibility and/or a mental health carve-out plan were excluded. Other therapies were not precluded during the study period so PP cohort patients could have had claims for OAT or a LAI antipsychotic other than PP at any point before or after index.

The OAT cohort was identified from among patients with no claims for PP between 2009 and 2012, in order to create mutually exclusive cohorts at index. Patients with two or more prescription claims for the same oral atypical antipsychotic (aripiprazole, asenapine, iloperidone, lurasidone, olanzapine [including combination olanzapine/fluoxetine], paliperidone, quetiapine, risperidone, or ziprasidone) on different dates, no more than 90 days apart, during the cohort selection period of July 2009 through December 2011 were identified. The earliest such claim was set as the index date (day 1). Patients with claims for the index antipsychotic in the 6 months pre-index were excluded so that all patients would be newly starting treatment with the index OAT on the index date. The continuous enrollment, age, diagnostic criteria, Medicare dual eligibility and carve-out criteria for the OAT cohort were the same as described above for the PP cohort. Use of PP by patients in the OAT cohort was precluded as a function of sample selection, but patients in the OAT cohort could have used non-index OATs or LAI antipsychotics other than PP at any point before or after index.

\subsection{Study Variables}

The primary outcomes for this study were differences in healthcare costs and healthcare utilization when OAT and PP cohorts were compared. Healthcare costs were identified by type of service and were calculated in two ways: all-cause and mental-health related. Mental-health-related costs were identified based on the primary/first diagnosis coded (ICD-9-CM 290.xx-319.xx) on each claim for outpatient and inpatient services and by pharmacy claims for all classes of psychiatric medications. Monthly costs in each of the two categories were calculated in 30-day increments, starting from the index date (day 1), such that month 1 costs were those incurred from days 1 through day 30 , month 2 costs were those incurred from day 31 through day 60, etcetera. All costs were inflation adjusted to 2012 US dollars using the Medical Care Component of the Consumer Price Index.
Healthcare utilization was similarly defined in two ways: all-cause and mental-health related. Binary measures of any utilization, as well as a count of the number of services incurred, were computed for inpatient admissions, emergency department visits, outpatient office visits, and other outpatient services. Monthly utilization rates were calculated in 30-day increments, starting from the index date, as described above for costs.

\subsection{Study Analyses}

In order to account for potential time-varying confounding that may occur across cohorts, multivariate analyses using MSM were conducted. MSM is a weighted repeated measures analysis with treatment as a time-varying covariate. It differs from the typical intent-to-treat modeling approach in that the MSM accounts for the fact that treatment decisions occur not only prior to index but over time. It also helps control for events that occur during the study period that may impact both post-index treatment decisions and outcomes of interest (e.g., hospitalization) that may be evident in claims data and can influence both subsequent treatment and outcomes.

MSM based on the Faries et al. methodology [20] were conducted to assess cost and utilization outcomes, while controlling for differences between cohorts and over time. Weights based on IPTW were calculated in a multi-step process. First, two weights were calculated for each patient using logistic regression. The clinical characteristics in Table 1 , measured monthly over the study period, were time-varying covariates in the regressions and the demographics characteristics in Table 1, measured at index, were non time-varying covariates. The first weight adjusted for treatment with PP (no or yes), and the second weight, relevant only for the PP cohort, adjusted for whether treatment with PP discontinued (no or yes). These two weights were then merged to form a stabilized weight as recommended in Hernán et al. [21]. Very large stabilized weights can be problematic in the analysis because these outliers represent almost certain assignment to a particular group, hence patients with large weights $(>30)$ were excluded from the MSM analysis. The value of 30 was thought to represent a reasonable compromise and was in line with the value used in other similar research. Next, a weighted repeated measures analysis of outcomes was performed, using a generalized estimating equation (GEE) approach. The GEE analysis used link functions and error distributions appropriate for the type of outcome; for example, log link and gamma error distribution for costs. In this fashion, per-patient, per-month costs could be analyzed. The same set of covariates as the second logistic regression together with the two variates treatment with $\mathrm{PP}$ and discontinuation of PP were covariates in the GEE 
Table 1 Baseline demographic and clinical characteristics of the study sample

\begin{tabular}{|c|c|c|c|c|c|c|}
\hline \multirow[t]{2}{*}{ Characteristic } & \multirow[t]{2}{*}{ Time frame } & \multicolumn{2}{|c|}{$\begin{array}{l}\text { PP initiators } \\
N=984\end{array}$} & \multicolumn{2}{|c|}{$\begin{array}{l}\text { OAT initiators } \\
N=4199\end{array}$} & \multirow[t]{2}{*}{$p$ value } \\
\hline & & $N /$ mean & $\% / \mathrm{SD}$ & $N /$ mean & $\% / \mathrm{SD}$ & \\
\hline \multicolumn{7}{|l|}{ Demographics } \\
\hline Age (mean, SD) & Index date & 38.8 & 12.3 & 41.6 & 12.9 & $<0.001$ \\
\hline Male $(n, \%)$ & Index date & 573 & $58.2 \%$ & 2049 & $48.8 \%$ & $<0.001$ \\
\hline Race/ethnicity $(n, \%)$ & Index date & & & & & \\
\hline White & & 377 & $38.3 \%$ & 1789 & $42.6 \%$ & 0.014 \\
\hline Black & & 511 & $51.9 \%$ & 1987 & $47.3 \%$ & 0.009 \\
\hline Other & & 96 & $9.8 \%$ & 423 & $10.1 \%$ & 0.765 \\
\hline Residence in $\operatorname{MSA}^{\mathrm{a}}(n, \%)$ & Index date & 734 & $74.6 \%$ & 3033 & $72.2 \%$ & 0.135 \\
\hline Capitated health plan $(n, \%)$ & Index date & 366 & $37.2 \%$ & 2224 & $53.0 \%$ & $<0.001$ \\
\hline \multicolumn{7}{|l|}{ Clinical characteristics } \\
\hline Type of schizophrenia $^{\mathrm{b}}(n, \%)$ & Most recent & & & & & \\
\hline All except schizoaffective & & 738 & $75.0 \%$ & 3236 & $77.1 \%$ & 0.168 \\
\hline Schizoaffective disorder & & 246 & $25.0 \%$ & 963 & $22.9 \%$ & 0.168 \\
\hline Charlson Comorbidity Index ${ }^{\mathrm{b}}$ (mean, SD) & Prior 6 months & 0.5 & 1.0 & 0.8 & 1.4 & $<0.001$ \\
\hline Mental health diagnoses ${ }^{\mathrm{b}}$ (mean, SD) & Prior 6 months & 3.2 & 2.3 & 3.4 & 2.3 & 0.014 \\
\hline Comorbidities $^{\mathrm{b}}(n, \%)$ & Prior 6 months & & & & & \\
\hline Depression & & 270 & $27.4 \%$ & 1572 & $37.4 \%$ & $<0.001$ \\
\hline Bipolar disorder & & 279 & $28.4 \%$ & 1436 & $34.2 \%$ & 0.001 \\
\hline Anxiety & & 57 & $5.8 \%$ & 222 & $5.3 \%$ & 0.527 \\
\hline Alcohol or substance abuse & & 122 & $12.4 \%$ & 539 & $12.8 \%$ & 0.711 \\
\hline Unique antipsychotic agents ${ }^{\mathrm{c}}(n, \%)$ & Prior 3 months & & & & & \\
\hline$<2$ unique agents & & 531 & $54.0 \%$ & 1197 & $28.5 \%$ & $<0.001$ \\
\hline$\geq 2$ unique agents & & 453 & $46.0 \%$ & 3002 & $71.5 \%$ & $<0.001$ \\
\hline Antipsychotic PDC ${ }^{c, d}$ (mean, SD) & Prior 3 months & 0.7 & 0.3 & 0.7 & 0.4 & $>0.999$ \\
\hline Other medication utilization $^{\mathrm{d}}(n, \%)$ & Prior 6 months & & & & & \\
\hline Long-acting injectable antipsychotics ${ }^{\mathrm{e}}$ & & 269 & $27.3 \%$ & 860 & $20.5 \%$ & $<0.001$ \\
\hline Antidepressants & & 518 & $52.6 \%$ & 2564 & $61.1 \%$ & $<0.001$ \\
\hline Mood stabilizers & & 417 & $42.4 \%$ & 1830 & $43.6 \%$ & 0.493 \\
\hline Anxiolytics & & 306 & $31.1 \%$ & 1622 & $38.6 \%$ & $<0.001$ \\
\hline Emergency department visit $(n, \%)$ & Prior 1 months & 438 & $44.5 \%$ & 2147 & $51.1 \%$ & $<0.001$ \\
\hline Inpatient admission $(n, \%)$ & Prior 3 months & 363 & $36.9 \%$ & 1796 & $42.8 \%$ & 0.001 \\
\hline Healthcare expenditures (mean, SD) & Prior 6 months & & & & & \\
\hline Total $^{\mathrm{f}}$ & & $\$ 19,431$ & $\$ 21,816$ & $\$ 22,070$ & $\$ 37,441$ & 0.004 \\
\hline Mental-health related $^{\mathrm{f}}$ & & $\$ 15,891$ & $\$ 19,773$ & $\$ 13,526$ & $\$ 17,946$ & 0.001 \\
\hline
\end{tabular}

Table presents metrics for Month 1 which started on the Index Date (day 1) and continued to day 30. Variables measured in the prior 1-6 months were compiled from claims prior to, but not including, the Index Date. The modeling employed the same variables measured for each of the 12 months of the study period (e.g., days $1-30$, days $31-60$, days $61-90$, etc.)

MSAs Metropolitan statistical areas, $O A T$ oral antipsychotic therapy, $P D C$ proportion of days covered, $P P$ paliperidone palmitate

${ }^{a}$ MSAs include cities with population $>50,000$ and urban areas with population $>100,000$. Non-MSAs are generally rural communities

${ }^{\mathrm{b}}$ Unique diagnoses codes measured from relevant ICD-9 codes listed on inpatient and outpatient services claims

${ }^{c}$ PDC $=$ days in past 90 days with any antipsychotic on hand (determined from medication claims), divided by 90

${ }^{\mathrm{d}}$ Measured from outpatient pharmacy claims and, for injectables, also from outpatient services claims

e Includes agents other than PP

${ }^{\mathrm{f}}$ Values are in 2012 US dollars 
analysis. The justification for this type of approach is that the ability to control for time-varying confounding accounted for the inter-cohort imbalances in covariates, which were observed across the 12-month study period.

In this study, every patient had a full 12 months of continuous enrollment so no patients discontinued from the study. However, patients could be non-adherent, discontinue or switch treatments, or discontinue treatment entirely. Because of the way the patients were selected, OAT patients could not switch to PP, which means that patients initially assigned PP could have discontinued it, switched to OAT, switched to an injectable other than PP, or stopped treatment altogether; and that patients initially assigned OAT could have discontinued their index OAT, switched to another OAT, switched to an injectable other than PP, or stopped treatment altogether. It was felt that this is too many alternatives to be practical to include in what is already a complex analysis. Restricting comparison to a two-group one (i.e., PP vs OAT) and with the way the coding was done (in particular that for discontinuation of $\mathrm{PP})$, it is assumed for the analysis that there is no discontinuation of OAT for those patients initially assigned to the OAT group, and that those patients in the PP group who discontinue with PP switch to OAT. In addition, there is a resemblance to an intent-to-treat approach in that any treatment other than PP or OAT is implicitly assumed to be OAT.

\section{Results}

The MarketScan Medicaid Multi-State Database contained 4778 patients initiating PP and 241,557 non-PP patients initiating OAT between the years 2009-2011. A higher percentage of patients in the PP cohort compared with the OAT cohort (66 vs $12 \%$ ) remained after applying the schizophrenia diagnosis criterion. After applying all inclusion and exclusion criteria, the final study sample contained 984 patients in the PP cohort and 4199 patients in the OAT cohort.

The baseline characteristics of the study sample are detailed in Table 1. Patients in the PP cohort were slightly younger than the OAT patients (38.8 vs 41.6 years, $p<0.001)$. There was a slightly higher percentage of males in the PP cohort (58.2 vs $48.8 \%, p<0.001)$. A lower percentage of patients in the PP cohort than the OAT cohort identified as White (38.3 vs $42.6 \%, p=0.014)$, and a higher percentage identified as Black (51.9 vs $47.3 \%$, $p=0.009$ ). Finally, a greater percentage of patients in the OAT cohort were members of a capitated health plan than patients in the PP cohort (53.0 vs $37.2 \%, p<0.001)$.

Clinically, the cohorts were similar with respect to the composition of patients with a primary schizophrenia subtype versus schizoaffective disorder (75.0 vs $77.1 \%$, $p=0.168$ ). The mean prior 6-month Charlson Comorbidity Index score was statistically significantly higher in the OAT cohort $(0.8$ vs $0.5, p<0.001)$. Patients in the OAT cohort also tended to have more unique mental health diagnoses (3.4 vs 3.2, $p=0.014$ ) and there was a higher frequency in this cohort of comorbidity with depression (37.4 vs $27.4 \%, p<0.001)$ and bipolar disorder (34.2 vs $28.4 \%, p=0.001)$.

In terms of recent treatment, patients in the OAT cohort were more frequently exposed to two or more unique antipsychotic agents in the previous 90 days (71.5 vs $46.0 \%, p<0.001$ ), and the mix of classes of psychotropic drugs differed between the two cohorts in the preceding 6 months. With respect to general medical care, there were a greater percentage of patients with an emergency department (ED) visit (51.1 vs $44.5 \%, p<0.001)$ and/or inpatient admission in the OAT cohort (42.8 vs $36.9 \%$, $p=0.001$ ), and their total healthcare expenditures tended to be higher (US $\$ 22,070$ vs US $\$ 19,431, p=0.004$ ). At baseline, expenditures for mental-health-related care were higher in the PP cohort (US\$15,891 vs US\$13,526, $p=0.001$ ).

After creation of stabilized weights for the MSM analysis and review of their distribution, it was determined that weights with a value $>30$ were outliers, and that observations with these weights should be excluded. A total of 87 patients, 20 of whom were in the PP cohort, had outlier stabilized weights and hence had observations excluded from the MSM analysis of cost and utilization outcomes.

After MSM adjustment to control for differences between cohorts, the mean cost differential between cohorts with respect to total and component costs (all-cause and mental-health related) was estimated (Table 2). Monthly prescription drug costs for patients in the PP cohort were higher than costs for patients in the OAT cohort, with respect to both all-cause pharmacy costs (US\$1004; $95 \%$ confidence interval [CI] 986-1021, $p<0.0001$ ) and mental-health-related costs (US\$1019; $95 \%$ CI 1005-1034, $p<0.0001)$. On the other hand, costs for other components of care, including inpatient and outpatient medical treatment, were lower in the PP cohort than the OAT cohort. Costs for inpatient services were lower, both in terms of all-cause costs (-US\$234; $95 \%$ CI -362 to $-107, p=0.0003$ ) and costs specific to mentalhealth-related care ( $-\mathrm{US} \$ 271 ; 95 \% \mathrm{CI}-355$ to -186 , $p<0.0001$ ). Similarly, costs for outpatient services, driven largely by costs other than office visits, were lower for the patients in the PP cohort, when considering all-cause costs ( $-\mathrm{US} \$ 336 ; 95 \% \mathrm{CI}-382$ to $-290, p<0.0001$ ) and those costs specific to mental-health care (-US\$286; $95 \%$ CI -318 to $-254, p<0.0001)$. 
Table 2 Mean adjusted permonth per-patient cost differential associated with PP utilization over 12 months postindex

\begin{tabular}{|c|c|c|c|c|}
\hline \multirow[t]{2}{*}{ Cost parameter } & \multirow[t]{2}{*}{ Estimate } & \multicolumn{2}{|c|}{ Wald $95 \%$ confidence limits } & \multirow[t]{2}{*}{ ProbChiSq } \\
\hline & & Lower & Upper & \\
\hline \multicolumn{5}{|l|}{ All-cause costs } \\
\hline Inpatient costs & -234.19 & -361.70 & -106.67 & 0.0003 \\
\hline Emergency department costs & -5.14 & -11.64 & 1.35 & 0.1208 \\
\hline Outpatient costs & -335.89 & -382.11 & -289.67 & $<0.0001$ \\
\hline Outpatient office visit costs & -0.55 & -2.13 & 1.02 & 0.4922 \\
\hline Other outpatient costs & -330.19 & -375.29 & -285.09 & $<0.0001$ \\
\hline Pharmacy costs & 1003.65 & 986.21 & 1021.09 & $<0.0001$ \\
\hline Total costs ${ }^{\mathrm{a}}$ & 433.58 & 297.88 & 569.27 & $<0.0001$ \\
\hline \multicolumn{5}{|l|}{ Mental-health-related costs ${ }^{\mathrm{b}}$} \\
\hline Inpatient costs & -270.56 & -354.67 & -186.45 & $<0.0001$ \\
\hline Emergency department costs & -0.22 & -2.79 & 2.35 & 0.8658 \\
\hline Outpatient costs & -285.94 & -317.70 & -254.17 & $<0.0001$ \\
\hline Outpatient office visit costs & 2.81 & 2.10 & 3.52 & $<0.0001$ \\
\hline Other outpatient costs & -288.52 & -320.12 & -256.93 & $<0.0001$ \\
\hline Pharmacy costs & 1019.30 & 1004.50 & 1034.10 & $<0.0001$ \\
\hline Total costs ${ }^{\mathrm{a}}$ & 462.80 & 373.95 & 551.65 & $<0.0001$ \\
\hline
\end{tabular}

Values are in 2012 US dollars

Reflects the mean monthly cost differential for patient months with PP compared to without PP, adjusted for the covariates shown in Table 1

$P P$ paliperidone palmitate

${ }^{\text {a }}$ Components do not sum to total costs because each cost parameter was modeled separately

b Includes claims with a primary diagnosis of ICD-9 290.xx-319.xx and pharmacy claims for all classes of psychiatric medications
Thus, approximately $55 \%$ of the mental-health-related prescription drug cost premium associated with PP was offset by lower costs of mental-health-related inpatient and outpatient care for these patients, producing a mean monthly total cost differential of US\$434 (95\% CI $298-569, p<0.0001)$ when accounting for all-cause costs or US $\$ 463$ (95\% CI 374-552, $p<0.0001$ ), accounting for mental-health-related costs.

Across the follow-up period, the use of PP as compared with OAT was associated with lower risk of healthcare resource utilization, on a per-month, per-patient basis (Table 3). For the PP cohort, the risk of an inpatient hospital admission was statistically significantly lower for both all-cause (by $36 \%$; $95 \%$ CI $40-30, p<0.0001$ ) and mental-health-related utilization (by $38 \%$; $95 \%$ CI 43-32, $p<0.0001)$. The risk of an ED visit for all causes was also lower (by $18 \%$; $95 \%$ CI $21-15, p=0.0134$ ).

\section{Discussion}

These results indicate that treatment with PP was associated with significantly lower inpatient admissions, lower inpatient costs, and lower outpatient costs compared with OAT treatment, which partially offset over half of the incremental drug acquisition costs. This information should prove valuable to healthcare decision makers and providers when selecting an appropriate treatment for patients with schizophrenia. The selection would be based on many factors, including effectiveness and total cost of care, which is comprised of drug acquisition cost and the cost of outcomes (i.e., hospitalization due to relapse, ED visits), both in terms of healthcare dollars and non-monetary costs (i.e., disruption to patients and caregivers).

The methods employed to control for differences between cohorts, both at index and over the follow-up period of the analysis, included IPTW and MSM. Given certain demographic, economic, and clinical differences between the two cohorts at baseline, such as a significantly higher incidence of mood disorder in the OAT cohort and higher mental-health-related costs in the 6 months pre-index, it was important to use this type of statistical methodology to answer the present research questions. The changing nature of certain variables over time may exert an influence on both treatment selection and measured outcomes, justifying the need to control for such time-dependent covariates with appropriate methodologies such as MSM. Studies that fail to account for the effect of time-dependent covariates may present biased results. 
Table 3 Adjusted per-month per-patient utilization risk ratios associated with PP utilization over 12 months post-index

\begin{tabular}{|c|c|c|c|c|}
\hline \multirow[t]{2}{*}{ Cost parameter } & \multirow[t]{2}{*}{ Estimate } & \multicolumn{2}{|c|}{ Wald $95 \%$ confidence limits } & \multirow[t]{2}{*}{ ProbChiSq } \\
\hline & & Lower & Upper & \\
\hline \multicolumn{5}{|l|}{ All-cause utilization } \\
\hline Any inpatient admission & 0.6441 & 0.5954 & 0.6967 & $<0.0001$ \\
\hline Any emergency department visit & 0.8228 & 0.7892 & 0.8468 & 0.0134 \\
\hline \multicolumn{5}{|l|}{ Mental health-related utilization ${ }^{\mathrm{a}}$} \\
\hline Any inpatient admission & 0.6235 & 0.5731 & 0.6779 & $<0.0001$ \\
\hline Any emergency department visit & 1.0539 & 0.9491 & 1.1587 & 0.1883 \\
\hline
\end{tabular}

Consistent with the findings of the current study, several studies conducted in the US and various countries worldwide have shown the incremental benefit of PP over OAT and other LAIs. Kozma et al., for example, reported a significant reduction in hospitalizations for patients with schizophrenia treated with PP compared with placebo and from prior to the trial to the end of the open-label phase of a double-blind randomized controlled trial [14]. In Sweden, a Markov model demonstrated the economic benefits of PP compared with long-acting formulations of risperidone and olanzapine [22]. Other studies have similarly demonstrated the initial promise of PP, in terms of reduced rates of relapse, medical costs, healthcare utilization, and qualityadjusted life-years [13, 19, 23, 24].

The findings from previous real-world studies comparing outcomes among Medicaid patients with schizophrenia treated with PP and patients prescribed OAT are consistent with the findings presented from this study [11, 25]. A Missouri Medicaid study, for example, reported a $37 \%$ reduction in the adjusted odds ratio for all-cause ED visits (AOR: 0.63), and lower adjusted odds ratio for all-cause and mental health-related hospitalizations in a propensityscore matched cohort of patients administered PP and patients prescribed OATs, with the ED difference at a statistically significant level [11]. While the differences in rate reductions between $\mathrm{PP}$ and OAT cohorts in our study are more pronounced (particularly with respect to the reduction of inpatient admissions) compared with the Missouri Medicaid study, they are directionally similar and provide further evidence of the benefit of PP in improving healthcare resource utilization-related outcomes.

This study's findings are also consistent with those from a claims-based study of Veterans Heath Administration patients with schizophrenia which revealed that patients treated with PP had lower mean inpatient costs (US\$18,560 vs US\$31,505, $p=0.002)$, lower hospitalization rate (34 vs $53 \%, p<0.001$ ), and fewer average inpatient days (13.24 vs 24.18, $p=0.002$ ) when compared with matched patients treated with OAT. While mean drug costs were higher for PP patients (US\$10,063 vs US\$4167, $p<0.001$ ) than OAT patients, mean total healthcare costs were similar between the groups (US\$45,529 vs US\$52,569, $p=0.128$ ) [18].

Contrary to the findings of the current study, a metaanalysis of randomized clinical trial results showed no difference in the rate of relapse and hospitalization between LAIs and OAT [26]. It could be that clinical trials, while vital to understanding the efficacy of antipsychotic agents, are not especially well suited to comparing LAIs and OATs on outcomes that may be associated with medication adherence because researcher monitoring throughout controlled trials minimizes the possibility of treatment nonadherence [27]. Thus, observational studies such as the current study may be especially useful in understanding how agents like PP may impact treatment outcomes in realworld settings. Consistent with this study's findings, a meta-analysis of mirror-image studies [28] as well as a meta-analysis of interventional and non-interventional studies using meta-regressions [19] suggested a benefit of LAI over OAT. This study adds to the existing body of observational research on this topic by employing MSM methodology that controls for time-dependent covariates to minimize bias.

Results of this study should be interpreted in light of certain limitations. Schizophrenia diagnoses were identified from healthcare claims coded for reimbursement rather than diagnostic purposes, thus misclassification was possible. Cohort assignment at index was based on healthcare claims that may be subject to measurement error. Similarly, administration of PP and initiation of OAT on the date listed on the claim was assumed, but could not be verified from the data. In terms of the present database, inpatient administration of PP could not be measured, thus some patients who appeared to have discontinuous therapy may have received injections while hospitalized. Additionally, costs for outpatient acquisition and administration of PP may have been included under pharmacy and/or outpatient services, depending on the requirements of the various state 
Medicaid plans included in the database. Medication dosing may have impacted costs and outcomes, but was not measured for either cohort as part of this study. Study results are reflective of a multi-state Medicaid population, but may not be generalizeable to non-Medicaid patient populations. Also, the assumptions about the data mentioned in the Methods section and those inherent to performing a complex MSM analysis could be leading to some biases in the results.

\section{Conclusion}

Due to the complexity of clinical management of schizophrenia, the propensity for patients to relapse and require re-hospitalization is high $[3,7]$. The prevention of relapse in schizophrenia remains a major public health challenge and continuous long-term antipsychotic therapy is considered essential to control symptoms and costly hospitalizations [8]. This study demonstrates that treatment with PP may address the propensity for relapse in this patient population, thereby reducing the rate of utilization of other healthcare services, while offsetting greater than half of its acquisition costs.

Acknowledgements Gilwan Kim of Truven Health Analytics assisted with manuscript revisions. Portions of this research were previously presented at the 27th Annual US Psychiatric and Mental Health Congress, September 20-23, 2014, Orlando, FL, USA.

\section{Compliance with Ethical Standards}

Funding This study was funded by Janssen Scientific Affairs, LLC.

Conflicts of interest J. A. Pesa and E. Muser are employees of Janssen Scientific Affairs, LLC and are Johnson \& Johnson stockholders. L. B. Montejano, D. M. Smith and O. I. Meyers are employees of Truven Health Analytics, an independent healthcare data and research company that received funding from Janssen Scientific Affairs, LLC to conduct this study.

Ethical approval This study was conducted using a pre-existing database of de-identified healthcare claims and, as such, did not require institutional review board (IRB)/independent ethics committee (IEC) approval.

Open Access This article is distributed under the terms of the Creative Commons Attribution-NonCommercial 4.0 International License (http://creativecommons.org/licenses/by-nc/4.0/), which permits any noncommercial use, distribution, and reproduction in any medium, provided you give appropriate credit to the original author(s) and the source, provide a link to the Creative Commons license, and indicate if changes were made.

\section{References}

1. McGuire TG. Measuring the economic costs of schizophrenia. Schizophr Bull. 1991;17(3):375-88.
2. Young AS, Sullivan G, Burnam MA, Brook RH. Measuring the quality of outpatient treatment for schizophrenia. Arch Gen Psychiatry. 1998;55:611-7.

3. Wu EQ, Birnbaum HG, Shi L, et al. The economic burden of schizophrenia in the United States in 2002. J Clin Psychiatry. 2005;66(9):1122-9.

4. Desai PR, Lawson KA, Barner JC, Rascati KL. Identifying patient characteristics associated with high schizophrenia-related direct medical costs in community-dwelling patients. J Manag Care Pharm. 2013;19(6):468-77.

5. O'Malley AJ, Frank RG, Normand S-LT. Estimating cost-offsets of new medications: use of new antipsychotics and mental health costs for schizophrenia. Stat Med. 2011;30(16):1971-88.

6. Almond S, Knap M, Francois C, et al. Relapse in schizophrenia: costs, clinical outcomes and quality of life. Br J Psychiatry. 2004; 184:346-51.

7. Karve SJ, Panish JM, Dirani RG, et al. Health care utilization and costs among Medicaid-enrolled patients with schizophrenia experiencing multiple psychiatric relapses. Health Outcomes Res Med. 2012;3:183-94.

8. Chen EY, Hui CL, Dunn EL, et al. A prospective 3-year longitudinal study of cognitive predictors of relapse in first-episode schizophrenic patients. Schizophr Res. 2005;77:99-104.

9. Weiden PJ, Kozma C, Grogg A, et al. Partial compliance and risk of rehospitalization among California Medicaid patients with schizophrenia. Psychiatr Serv. 2004;55:886-91.

10. Brown JD, Barrett A, Ireys H, et al: Evidence-based practices for Medicaid beneficiaries with Schizophrenia and Biopolar disorder. No. 7490. Mathematica Policy Research. 2012. http:// aspe.hhs.gov/daltcp/reports/2012/ebpsbd.shtml. Accessed 6 Nov 2013.

11. Morrato EH, Parks J, Campagna EJ. Comparative effectiveness of injectable paliperidone palmitate versus oral atypical antipsychotics: early postmarketing evidence. J Compar Effect Res. 2014 (Epub ahead of print).

12. Einarson TR, Geitona M, Chaidemenos A, et al. Pharmacoeconomic analysis of paliperidone palmitate for treating schizophrenia in Greece. Ann Gen Psychiatry. 2012;11(1):18.

13. Achilla E, McCrone P. The cost-effectiveness of long-acting/ extended-release antipsychotics for the treatment of schizophrenia: a systematic review of economic evaluations. Appl Health Econ Health Policy. 2013;11(2):95-106.

14. Kozma CM, Slaton T, Dirani R, et al. Changes in schizophreniarelated hospitalization and ER use among patients receiving paliperidone palmitate: results from a clinical trial with a 52-week open label extension. Curr Med Res Opin. 2011;27: $1603-11$.

15. Garcia-Ruiz AJ, Perez-Costillias L, Montesinos AC, et al. Costeffectiveness analysis of antipsychotics in reducing schizophrenia relapses. Health Econ Rev. 2012;2:8-19.

16. Edwards NC, Rupnow MF, Pashos CL, et al. Cost-effectiveness model of long-acting risperidone in schizophrenia in the US. PharmacoEconomics. 2005;23:299-314.

17. Geitona M, Kousoulakou H, Ollandezos M, et al. Costs and effects of paliperidone extended release compared with alternative oral antipsychotic agents in patients with schizophrenia in Greece: a cost effectiveness study. Ann Gen Psychiatry. 2008;7:16-27.

18. Baser O, Xie L, Pesa J, Durkin M. Healthcare utilization and costs of veterans health administration patients with schizophrenia treated with paliperidone palmitate long-acting injection or oral atypical antipsychotics. J Med Econ. 2015;18(5):357-65.

19. Lafeuille MH, Dean J, Carter V, et al. Systematic review of longacting injectables versus oral atypical antipsychotics on hospitalization in schizophrenia. Curr Med Res Opin. 2014;30(8): $1643-55$. 
20. Faries D, Leon A, Haro J. Analysis of observational health care data using SAS. SAS Press. 2010.

21. Hernán M, Brumback B, Robins J. Estimating the causal effect of zidovudine on CD4 count with a marginal structural model for repeated measures. Stat Med. 2002;21:1689-709.

22. Mehnert A, Nicholl D, Pudas H, et al. Cost effectiveness of paliperidone palmitate versus risperidone long-acting injectable and olanzapine palmotate for the treatment of patients with schizophrenia in Sweden. J Med Econ. 2012;15 (5):844-61.

23. Zeidler J, Mahlich J, Greiner W, Heres S. Cost effectiveness of paliperidone palmitate for the treatment of schizophrenia in Germany. Appl Health Econ Health Policy. 2013;11(5):509-21.

24. Einarson TR, Vicente C, Zilbershtein R, et al. Pharmacoeconomics of depot antipsychotics for treating chronic schizophrenia in Sweden. Nord J Psychiatry. 2014;68(6):416-27.
25. Xiao X, Muser E, Lafeuille MH, et al. Impact of paliperidone palmitate versus oral atypical antipsychotics on healthcare outcomes in schizophrenia patients. J Comp Eff Res. 2015;14:1-14 (Epub ahead of print).

26. Kishimoto T, Robenzadeh A, Leucht C, Leucht S, Koichiro W, et al. Long-acting injectable vs oral antipsychotics for relapse prevention in schizophrenia: a meta-analysis of randomized trials. Schizophr Bull. 2014;40(1):192-213.

27. Kirson NY, Weiden PJ, Yermakov S, Huang W, Samuelson T, Offord SJ, et al. Efficacy and effectiveness of depot versus oral antipsychotcis in schizophrenia: synthesizing results across different research designs. J Clin Psychiatry. 2013;74:568-75.

28. Kishimoto T, Borenstein NM, Kane JM, Correll CU. Long-acting injectable versus oral antipsychotics in schizophrenia: a systematic review and meta anlaysis or mirro-image studies. J Clin Psychiatry. 2013;74(10):957-65. 